\title{
Continued Fractions and Digital Lines with Irrational Slopes
}

\author{
Hanna Uscka-Wehlou \\ Uppsala University, Department of Mathematics \\ Box 480, SE-751 06 Uppsala, Sweden \\ hania@wehlou.com \\ http://wehlou.com/hania/index.htm
}

\begin{abstract}
This paper expands on previous work on relationships between digital lines and continued fractions (CF). The main result is a parsimonious description of the construction of the digital line based only on the elements of the CF representing its slope and containing only simple integer computations. The description reflects the hierarchy of digitization runs, which raises the possibility of dividing digital lines into equivalence classes depending on the $\mathrm{CF}$ expansions of their slopes. Our work is confined to irrational slopes since, to our knowledge, there exists no such description for these, in contrast to rational slopes which have been extensively examined. The description is exact and does not use approximations by rationals. Examples of lines with irrational slopes and with very simple digitization patterns are presented. These include both slopes with periodic and non-periodic CF expansions, i.e. both quadratic surds and other irrationals.
\end{abstract}

Keywords: digital geometry, theory of digital lines, irrational slope, continued fraction, quadratic surd.

\section{Introduction}

\subsection{About This Paper}

The aim of the present paper is to solve the following problem: given the continued fraction (CF) expansion of the slope $a \in] 0,1[\backslash \mathbb{Q}$ of a straight line, how is the digitization of this line constructed? The description uses only the elements of the $\mathrm{CF}$ and is exact, i.e. does not use the commonly applied approximations by rationals. The method is based on simple integer computations that can be easily applied to computer programming.

This description forms the Main Result (Theorem 4 description by CFs). The theoretical basis for this article is Uscka-Wehlou (2007) [15. The main result there is recalled in Sect. 2] of the present paper (Theorem 1] description by the digitization parameters). It gives a description of digitization runs on all digitization levels for lines $y=a x$ where $a \in] 0,1[\backslash \mathbb{Q}$, which is based on digitization parameters defined in Def. 1 and the function $\operatorname{Reg}_{a}$ defined in Def. 2. 
Although Theorem 1 1 looks similar to Theorem 4, the former involves computations on irrational numbers, which is not the case in the latter.

The idea of the new description was to replace the heavy computations involved in the description by digitization parameters by simple computations on integers. In order to do that, the digitization parameters and the function $\operatorname{Reg}_{a}$ for each $a \in] 0,1[\backslash \mathbb{Q}$ were expressed by the elements of the CF expansion of $a$. The key role in this transform is played by the index jump function (Def. 3).

The main work leading to the successful translation of Theorem 1 into the CF description (Theorem 4) has been done in Theorems 2 and 3. The first one expresses the digitization parameters in terms of CFs and the second one does the same with the function $\operatorname{Reg}_{a}$. These results allowed us to replace the computationally challenging conditions and formulae for run lengths by equivalent conditions and formulae based on the elements of the CF expansion of $a$.

In general, it is hard to perform arithmetical operations on CFs; see Khinchin 1997 (p. 20 in [7). However, Def. 1 and Theorem 1 involve only the operations which form an exception to this rule. These operations are: finding the inverse to a $\mathrm{CF}$, finding the integer (fractional) part of the inverse to a $\mathrm{CF}$, and subtracting a CF from 1. The formula for the last operation is described in Lemmas 1 and 2, the others are clearly easy to perform. This made it possible to find the simple description formulated in Theorem 4 .

The computations on irrationals did not disappear during the translation of Theorem 1 into the CF version (Theorem 4). They were moved into the process of finding the CF expansion of the slope. For some slopes we are able to compute the CF expansions exactly, using mathematical methods; some examples will be shown in Sect. 4. for both algebraic and transcendental numbers. For other slopes we can use algorithms for finding CF expansions.

The present CF description of digital lines is similar to the formula of Markov and Venkov ([16], p. 67), but since their method was not meant for descriptions of digital lines, it does not reflect the hierarchical structure of digitization runs on all levels, which our method does. This permits the grouping of digital lines into classes according to properties defined by the elements of the CF expansions of the slopes (to be presented in a forthcoming paper by the author).

The new method presented here is computationally simple, involving only easy computations with integers, excepting the algorithm for determining the CF expansion of the slope. The method applies to irrational slopes and gives the exact results instead of approximations by rationals. To the author's knowledge, there are no previous descriptions of digital lines with irrational slopes fulfilling all the criteria just mentioned, and reflecting the hierarchy of runs.

\subsection{Earlier Developments}

The use of CFs in modelling digital lines was discussed by R. Brons [3] as early as in 1974. Already then it was clear that the patterns generated in the digitization process of straight lines were related to the CF expansions of the slopes. However, the algorithm provided by Brons is only valid for rational slopes. 
Some other researchers describing the construction of digital lines with rational slopes in terms of CFs were J. P. Reveillès (1991) [11, K. Voss (1993: 153-157) [18] - the splitting formula, I. Debled (1995: 59-66) [5] - description by the Stern-Brocot tree, P. D. Stephenson (1998) [13] - an algorithmic solution, F. de Vieilleville and J.-O. Lachaud (2006) [17] - a combinatoric approach. See also the review of R. Klette and A. Rosenfeld from 2004 [8].

Irrational numbers have been less central in research on digital line construction, possibly because irrational slopes must appear not to have direct applications for computer graphics. Nevertheless, irrational numbers may play an important role in our understanding of computer graphics theory which has a basis in digital geometry.

A CF description of digital lines was presented by L. Dorst and R. P. W. Duin (1984) 6]. Although their solution can be applied to irrational slopes, it is formulated as an algorithm. Since it is not a mathematical theorem, it will not result in descriptions of digital lines as mathematical objects, or help research on their abstract properties.

L. D. Wu formulated in 1982 a theorem describing digital straightness. Proofs of this theorem based on CFs were published in 1991 independently by A. M. Bruckstein and K. Voss; see Klette and Rosenfeld (2004: 208-209) 8]. A. M. Bruckstein described digital straightness in 4. by a number of transformations preserving it. Some of these transformations were defined by CFs.

Some work on the subject has also been done outside digital geometry and computer graphics, however, the solutions obtained in other fields do not reflect the hierarchical structure of digitization runs, which is an important feature of digital lines as mathematical objects.

For example, as far back as in 1772, astronomer Johan III Bernoulli applied the CF expansion of $a$ to the solution of the problem of describing the sequence $(\lfloor n a\rfloor)_{n \in \mathbb{N}^{+}}$for an irrational $a$. The problem is clearly equivalent to finding the digitization of $y=a x$. Bernoulli failed to provide any proofs. Venkov catalogued the entire history of the problem and its solution (including the solution by Markov from 1882) in [16] (pp. 65-71).

Stolarsky (1976) described in [14] applications of CFs to Beatty sequences.

Last but not least, we have to mention the research on Sturmian words, because this is very closely related to the research on digital lines with irrational slopes; see chapter 2 in [9] (by J. Berstel and P. Séébold).

\section{Earlier Results by Uscka-Wehlou (2007) [15]}

This section presents results obtained by the author in [15. In the current paper, as in [15], we will discuss the digitization of lines with equations $y=a x$ where $a \in] 0,1[\backslash \mathbb{Q}$. Also here, like in $[15$, the standard Rosenfeld digitization (Rdigitization) is replaced by the $\mathrm{R}^{\prime}$-digitization. The $\mathrm{R}^{\prime}$-digitization of the line with equation $y=a x$ is equal to the R-digitization of $y=a x+\frac{1}{2}$. For the definitions of both digitizations see [15. The $\mathrm{R}^{\prime}$-digitization of $y=a x$ was obtained there using the following digitization parameters. 
Definition 1. For $y=a x$, where $a \in] 0,1[\backslash \mathbb{Q}$, the digitization parameters are:

$$
\sigma_{1}=\operatorname{frac}\left(\frac{1}{a}\right)
$$

and for all natural numbers $k>1$

$$
\left.\sigma_{k}=\operatorname{frac}\left(1 / \sigma_{k-1}^{\wedge}\right), \quad \text { where } \sigma_{k-1}^{\wedge}=\min \left(\sigma_{k-1}, 1-\sigma_{k-1}\right) \in\right] 0, \frac{1}{2}[\backslash \mathbb{Q} .
$$

For $j \in \mathbb{N}^{+}, \sigma_{j}$ and $\sigma_{j}^{\wedge}$ are the digitization parameters and modified digitization parameters of the digitization level $j$, respectively.

For each $a \in] 0,1\left[\backslash \mathbb{Q}\right.$, an auxiliary function $\operatorname{Reg}_{a}$ was introduced. This function gives for each $k \geq 2$ the number of all the digitization levels $i$, where $1 \leq i \leq k-1$, with digitization parameters fulfilling the condition $\sigma_{i}<\frac{1}{2}$.

Definition 2. For a given line with equation $y=a x$, where $a \in] 0,1[\backslash \mathbb{Q}$, we define a function $\operatorname{Reg}_{a}: \mathbb{N}^{+} \longrightarrow \mathbb{N}$ as follows:

$$
\operatorname{Reg}_{a}(k)= \begin{cases}0 & \text { if } k=1 \\ \sum_{i=1}^{k-1} \chi_{] 0,1 / 2[}\left(\sigma_{i}\right) & \text { if } k \in \mathbb{N}^{+} \backslash\{1\},\end{cases}
$$

where $\chi_{] 0,1 / 2[}$ is the characteristic function of the interval $] 0, \frac{1}{2}[$.

The digitization runs of level $k$ for $k \in \mathbb{N}^{+}$were defined recursively as sets of runs of level $k-1$ (if we define integer numbers as runs of level 0 ). We used to call $\operatorname{run}_{k}(j)$ for $k, j \in \mathbb{N}^{+}$a run of digitization level $k$. We used notation run $_{k}$ or in plural $\operatorname{runs}_{k}$, meaning $\operatorname{run}_{k}(j)$ for some $j \in \mathbb{N}^{+}$, or, respectively, $\left\{\operatorname{run}_{k}(i) ; i \in I\right\}$ where $I \in \mathcal{P}\left(\mathbb{N}^{+}\right)$. We also defined the length of a digitization run as its cardinality.

Function $\operatorname{Reg}_{a}$ defined in Def. 2 was very important in the description of the form of runs. It helped to recognize which kind of runs was the most frequent (also called main) on each level and which kind of runs was first, i.e., beginning in $(1,1)$. We showed that for a given straight line $l$ with equation $y=a x$, where $a \in] 0,1[\backslash \mathbb{Q}$, the $\mathrm{R}^{\prime}$-digitization of the positive half line of $l$ is the following subset of $\mathbb{Z}^{2}$ :

$$
D_{R^{\prime}}(l)=\bigcup_{j \in \mathbb{N}^{+}}\left\{\operatorname{run}_{1}(j) \times\{j\}\right\} .
$$

This was a part of the main result achieved in [15].

The main theorem of [15] was a formalization of the well-known conditions the digitization runs fulfill. On each level $k$ for $k \geq 1$ we have short runs $S_{k}$ and long runs $L_{k}$, which are composed of the runs of level $k-1$. Only one type of the runs (short or long) on each level can appear in sequences, the second type always occurs alone.

Notation. In the present paper we will use the notation $S_{k}^{m} L_{k}, L_{k} S_{k}^{m}, L_{k}^{m} S_{k}$ and $S_{k} L_{k}^{m}$, where $m=\left\lfloor 1 / \sigma_{k}^{\wedge}\right\rfloor-1$ or $m=\left\lfloor 1 / \sigma_{k}^{\wedge}\right\rfloor$, when describing the form of digitization runs $_{k+1}$. For example, $S_{k}^{m} L_{k}$ means that the $\operatorname{run}_{k+1}$ we are talking about consists of $m$ short runs ${ }_{k}\left(\right.$ abbreviated $S_{k}$ ) and one long run (abbreviated $^{2}$ 
$\left.L_{k}\right)$ in this order, so it is a $\operatorname{run}_{k+1}$ with the most frequent element short. The length of such a run ${ }_{k+1}$, being its cardinality, i.e., the number of runs ${ }_{k}$ contained in it, is then equal to $m+1$. We will also use the notation $\left\|S_{k+1}\right\|$ and $\left\|L_{k+1}\right\|$ for the length of the short resp. long runs $_{k+1}$.

We will use the following reformulation of the main result from [15].

Theorem 1 (Main Result in [15]; description by the digitization parameters). For a straight line with equation $y=a x$, where $a \in] 0,1[\backslash \mathbb{Q}$, we have $\left\|S_{1}\right\|=\left\lfloor\frac{1}{a}\right\rfloor,\left\|L_{1}\right\|=\left\lfloor\frac{1}{a}\right\rfloor+1$, and the forms of runs $_{k+1}$ (form_run fort $_{k}$ ) for $k \in \mathbb{N}^{+}$are as follows:

form_run $_{k+1}=\left\{\begin{array}{lll}S_{k}^{m} L_{k} & \text { if } \operatorname{Reg}_{a}(k+1)=\operatorname{Reg}_{a}(k)+1 & \text { and } \operatorname{Reg}_{a}(k) \text { is even } \\ S_{k} L_{k}^{m} & \text { if } \operatorname{Reg}_{a}(k+1)=\operatorname{Reg}_{a}(k) & \text { and } \operatorname{Reg}_{a}(k) \text { is even } \\ L_{k} S_{k}^{m} & \text { if } \operatorname{Reg}_{a}(k+1)=\operatorname{Reg}_{a}(k)+1 & \text { and } \operatorname{Reg}_{a}(k) \text { is odd } \\ L_{k}^{m} S_{k} & \text { if } \operatorname{Reg}_{a}(k+1)=\operatorname{Reg}_{a}(k) & \text { and } \operatorname{Reg}_{a}(k) \text { is odd, }\end{array}\right.$

where $m=\left\lfloor\frac{1}{\sigma_{k}^{\wedge}}\right\rfloor-1$ if the $\operatorname{run}_{k+1}$ is short and $m=\left\lfloor\frac{1}{\sigma_{k}^{\wedge}}\right\rfloor$ if the $\operatorname{run}_{k+1}$ is long. The function $\operatorname{Reg}_{a}$ is defined in Def. Q, and $\sigma_{k}$ for $k \in \mathbb{N}^{+}$in Def. 1 .

Theorem 1 shows exactly how to find the $\mathrm{R}^{\prime}$-digitization of the positive half line $y=a x$ for $a \in] 0,1[\backslash \mathbb{Q}$. We get the digitization by calculating the digitization parameters and proceeding step by step, recursively. The knowledge about the kind of the first run on each level allows us go as far as we want in the digitization. The only problem was in the heavy computation of the $\sigma$-parameters, but this will be solved now, in Sect. 3 .

\section{Main Result}

Before presenting the description of the digitization, we provide a brief introduction on CFs. For more details see e.g. [7.

Let $a$ be an irrational number. The following algorithm gives the regular (or simple) CF for $a$, which we denote by $\left[a_{0} ; a_{1}, a_{2}, a_{3}, \ldots\right]$. We define a sequence of integers $\left(a_{n}\right)$ and a sequence of real numbers $\left(\alpha_{n}\right)$ by:

$$
\alpha_{0}=a ; \quad a_{n}=\left\lfloor\alpha_{n}\right\rfloor \text { and } \alpha_{n}=a_{n}+\frac{1}{\alpha_{n+1}} \text { for } n \geq 0 .
$$

Then $a_{n} \geq 1$ and $\alpha_{n}>1$ for $n \geq 1$. The natural numbers $a_{0}, a_{1}, a_{2}, a_{3}, \ldots$ are called the elements of the CF. They are also called the terms of the CF, see p. 20 in [1]; or partial quotients, see p. 40 in [16]. We use the word elements, following Khinchin (p. 1 in [7]).

If $a$ is irrational, so is each $\alpha_{n}$, and the sequences $\left(a_{n}\right)$ and $\left(\alpha_{n}\right)$ are infinite. A CF expansion exists for all $a \in \mathbb{R}$ and is unique if we impose an additional condition that the last element (if $a$ is rational) cannot be 1; see [7, p. 16 .

The following two lemmas concern subtracting CFs from 1.

Lemma 1. Let $b_{i} \in \mathbb{N}^{+}$for all $i \in \mathbb{N}^{+}$and $b_{1} \geq 2$. Then

$$
1-\left[0 ; b_{1}, b_{2}, b_{3}, \ldots\right]=\left[0 ; 1, b_{1}-1, b_{2}, b_{3}, \ldots\right] .
$$


Proof. Let $b=\left[0 ; b_{1}, b_{2}, b_{3}, \ldots\right]$ and $b_{1} \geq 2$. Then $\frac{1}{b}=\left[b_{1} ; b_{2}, b_{3}, \ldots\right]$ and we get $1-b=1 /\left(1+1 /\left(\frac{1}{b}-1\right)\right)=1 /\left(1+1 /\left(\left[b_{1} ; b_{2}, b_{3}, \ldots\right]-1\right)\right)=\left[0 ; 1, b_{1}-1, b_{2}, \ldots\right]$.

Lemma 2. If $a_{i} \in \mathbb{N}^{+}$for all $i>1$, then $1-\left[0 ; 1, a_{2}, a_{3}, \ldots\right]=\left[0 ; a_{2}+1, a_{3}, \ldots\right]$. Proof. Put $b_{1}-1=a_{2}, b_{2}=a_{3}, b_{3}=a_{4}, \ldots, b_{i}=a_{i+1}, \ldots$ in Lemma 1.

Because clearly $\left[0 ; 1, a_{2}, a_{3}, \ldots\right]>\frac{1}{2}$ for all sequences $\left(a_{2}, a_{3}, \ldots\right)$ of positive integers, Lemma 2 illustrates the modification operation for the $\sigma$-parameters according to Def. 1. This leads us to define the following index jump function, which will allow us describe the digitization in terms of CFs.

Definition 3. For each $a \in] 0,1\left[\backslash \mathbb{Q}\right.$, the index jump function $i_{a}: \mathbb{N}^{+} \rightarrow \mathbb{N}^{+}$ is defined by $i_{a}(1)=1, i_{a}(2)=2$ and $i_{a}(k+1)=i_{a}(k)+1+\delta_{1}\left(a_{i_{a}(k)}\right)$ for $k \geq 2$, where $\delta_{1}(x)=\left\{\begin{array}{l}1, x=1 \\ 0, x \neq 1\end{array}\right.$ and $a_{1}, a_{2}, \ldots \in \mathbb{N}^{+}$are the CF elements of $a$.

The following theorem translates Def. 1 into the language of CFs. It is a very important step on the way of translating our earlier results into a simple CF description. This will also serve as a springboard for future research on the connection between the sequences of the consecutive digitization parameters for given $a \in] 0,1\left[\backslash \mathbb{Q}\right.$ and the iterations of the Gauss Map $G(a)=\operatorname{frac}\left(\frac{1}{a}\right)$.

Theorem 2. Let $a \in] 0,1\left[\backslash \mathbb{Q}\right.$ and $a=\left[0 ; a_{1}, a_{2}, \ldots\right]$. For the digital straight line with equation $y=a x$, the digitization parameters as defined in Def. 1 are

$$
\sigma_{k}=\left[0 ; a_{i_{a}(k+1)}, a_{i_{a}(k+1)+1}, \ldots\right] \text { for } k \geq 1
$$

where $i_{a}$ is the index jump function defined in Def. 3 .

Proof. The proof will be by induction. For $k=1$, the statement is $\sigma_{1}=$ $\left[0 ; a_{2}, a_{3}, \ldots\right]$, because $i_{a}(2)=2$. From Def. 1 and because $a=\left[0 ; a_{1}, a_{2}, \ldots\right]$, we have $\sigma_{1}=\operatorname{frac}\left(\frac{1}{a}\right)=\left[0 ; a_{2}, a_{3}, \ldots\right]$, so the induction hypothesis for $k=1$ is true. Let us now suppose that $\sigma_{k}=\left[0 ; a_{i_{a}(k+1)}, a_{i_{a}(k+1)+1}, \ldots\right]$ for some $k \geq 1$. We will show that this implies that $\sigma_{k+1}=\left[0 ; a_{i_{a}(k+2)}, a_{i_{a}(k+2)+1}, \ldots\right]$. From Def. 3 we have $i_{a}(k+2)=i_{a}(k+1)+1+\delta_{1}\left(a_{i_{a}(k+1)}\right)$. According to Def. 1 ] $\sigma_{k+1}=\operatorname{frac}\left(1 / \sigma_{k}^{\wedge}\right)$. We get two cases:

- $a_{i_{a}(k+1)} \neq 1\left(\right.$ thus $\left.\delta_{1}\left(a_{i_{a}(k+1)}\right)=0\right)$. This means that $\sigma_{k}<\frac{1}{2}$, so $\sigma_{k}^{\wedge}=\sigma_{k}$. We get the statement, because $\sigma_{k+1}=\operatorname{frac}\left(1 / \sigma_{k}\right)=\left[0 ; a_{i_{a}(k+1)+1}, \ldots\right]=\left[0 ; a_{i_{a}(k+2)}, \ldots\right]$.

- $a_{i_{a}(k+1)}=1\left(\delta_{1}\left(a_{i_{a}(k+1)}\right)=1\right)$. This means that $\sigma_{k}>\frac{1}{2}$, so $\sigma_{k}^{\wedge}=1-\sigma_{k}$. Lemma 2 and Def. 3 give us the statement, because $\sigma_{k+1}=\operatorname{frac}\left(1 /\left(1-\sigma_{k}\right)\right)=\left[0 ; a_{i_{a}(k+1)+2}, \ldots\right]=\left[0 ; a_{i_{a}(k+2)}, \ldots\right]$.

This completes the proof.

In order to get a $\mathrm{CF}$ description of the digitization, we will express the function $\operatorname{Reg}_{a}$ (determining the form of the digitization runs on all the levels) using the 
function $i_{a}$ defined in Def. 3. The translation of Def. 2 into the following CF version results in a very simple relationship between the complicated $\operatorname{Reg}_{a}$ and the simple $i_{a}$. It is a very important step in translating Theorem 1 into a CF version.

Theorem 3. For a given $a \in] 0,1[\backslash \mathbb{Q}$, there is the following connection between the corresponding functions $\operatorname{Reg}_{a}$ and $i_{a}$. For each $k \in \mathbb{N}^{+}$

$$
\operatorname{Reg}_{a}(k)=2 k-i_{a}(k+1) .
$$

Proof. For $k=1$ a direct check gives the equality. Let us assume that $\operatorname{Reg}_{a}(k)=$ $2 k-i_{a}(k+1)$ for some $k \geq 1$. We will show that this implies $\operatorname{Reg}_{a}(k+1)=$ $2 k+2-i_{a}(k+2)$, which will, by induction, prove our statement.

It follows from Def. 2, that for $k \geq 1$

$$
\operatorname{Reg}_{a}(k+1)=\operatorname{Reg}_{a}(k)+\chi_{] 0,1 / 2[}\left(\sigma_{k}\right) .
$$

Moreover, according to Def. 3, for $k \geq 1$

$$
i_{a}(k+2)=i_{a}(k+1)+1+\delta_{1}\left(a_{i_{a}(k+1)}\right) .
$$

Putting (8) and (9) in the induction hypothesis for $k+1$, we see that we have to show the following:

$$
\operatorname{Reg}_{a}(k)+\chi_{] 0,1 / 2[}\left(\sigma_{k}\right)=2 k+2-\left(i_{a}(k+1)+1+\delta_{1}\left(a_{i_{a}(k+1)}\right)\right) .
$$

Due to the induction hypothesis for $k$, it is enough to show that for all $k \geq 1$

$$
\chi_{] 0,1 / 2[}\left(\sigma_{k}\right)=1-\delta_{1}\left(a_{i_{a}(k+1)}\right) .
$$

To prove this, we use Theorem 2, which says that $\sigma_{k}=\left[0 ; a_{i_{a}(k+1)}, \ldots\right]$ :

$$
\begin{aligned}
\chi_{] 0,1 / 2[}\left(\sigma_{k}\right)=1 & \Leftrightarrow\left[0 ; a_{i_{a}(k+1)}, a_{i_{a}(k+1)+1}, \ldots\right]<\frac{1}{2} \\
& \Leftrightarrow a_{i_{a}(k+1)} \neq 1 \Leftrightarrow 1-\delta_{1}\left(a_{i_{a}(k+1)}\right)=1 .
\end{aligned}
$$

This completes the proof.

Now we are ready to formulate our main theorem. The theorem is more parsimonious from a computational standpoint than Theorem 1, because the function $i_{a}$ is very simple and contains only computations with integers. This is an important advantage for efficient computer program development. The entire description uses only one function: the index jump function.

Theorem 4 (Main Result; description by CFs). Let $a \in] 0,1[\backslash \mathbb{Q}$ and $a=\left[0 ; a_{1}, a_{2}, \ldots\right]$. For the digital straight line with equation $y=a x$, we have $\left\|S_{1}\right\|=a_{1},\left\|L_{1}\right\|=a_{1}+1$, and the forms of runs $_{k+1}$ (form_run fort $_{k}$ ) for $k \in \mathbb{N}^{+}$ are as follows:

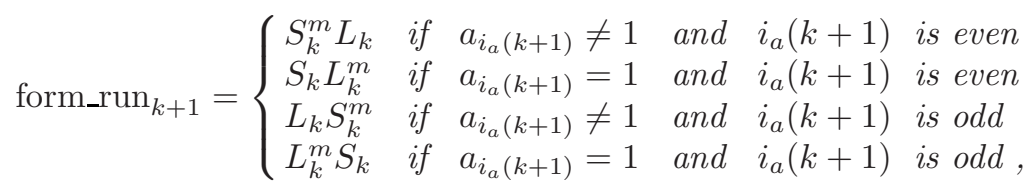


where $m=b_{k+1}-1$ if the $\operatorname{run}_{k+1}$ is short and $m=b_{k+1}$ if the $\operatorname{run}_{k+1}$ is long. The function $i_{a}$ is defined in Def. 3 and $b_{k+1}=a_{i_{a}(k+1)}+\delta_{1}\left(a_{i_{a}(k+1)}\right) a_{i_{a}(k+1)+1}$.

Figure 1 illustrates the connection between the hierarchy of runs (the first five levels), the index jump function and the digitization parameters for $y=a x$, where $a=\left[0 ; 1,2,1,1,3,1,1, a_{8}, a_{9}, \ldots\right]$ for some $a_{8}, a_{9}, \ldots \in \mathbb{N}^{+}$.

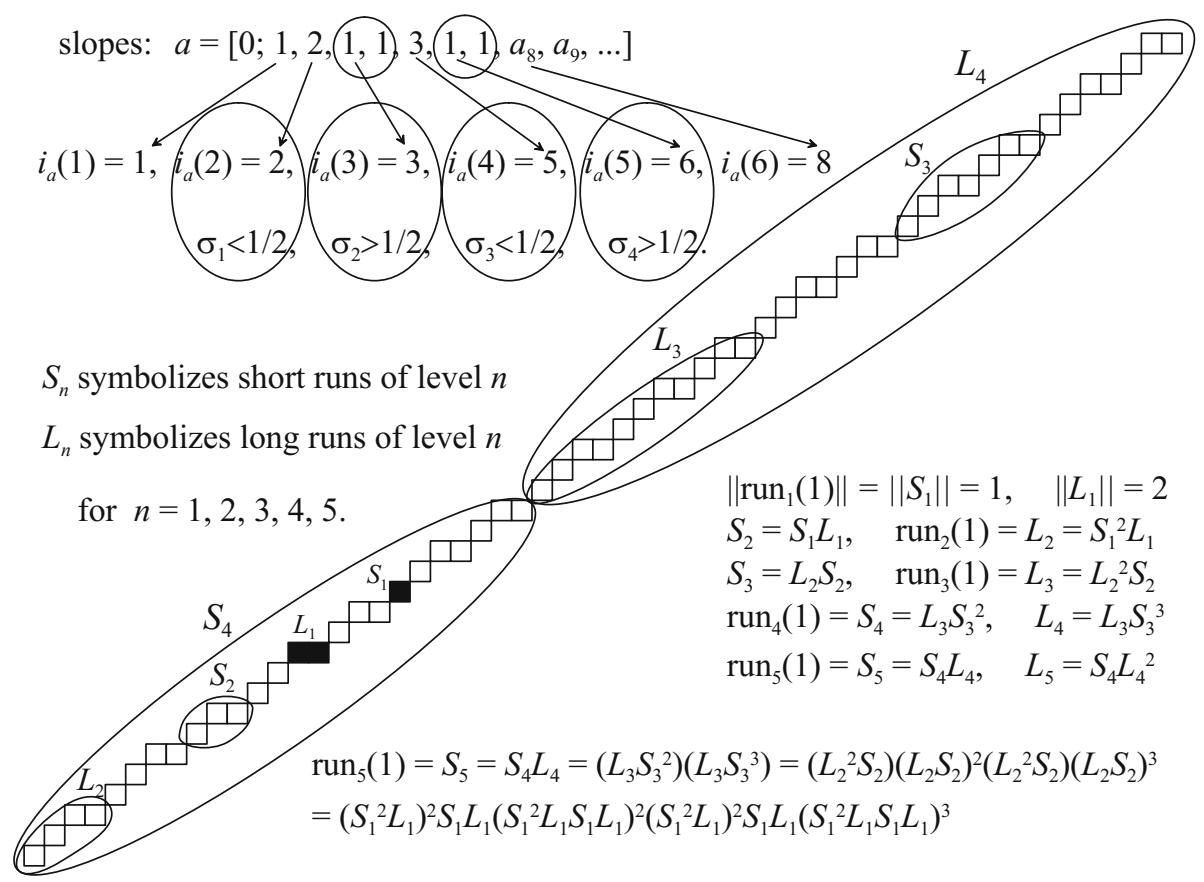

Fig. 1. The index jump function, digitization parameters and hierarchy of runs

Proof. Theorem 4 follows from Theorems 1, 2 and 3, From Theorem 1 we know that the length of the short $\mathrm{runs}_{k+1}$ is $\left\lfloor\frac{1}{\sigma_{k}^{\star}}\right\rfloor$. According to Theorem 2, $\sigma_{k}=$ $\left[0 ; a_{i_{a}(k+1)}, a_{i_{a}(k+1)+1}, \ldots\right]$. We have to consider two possible cases:

- $a_{i_{a}(k+1)}>1$. This means that $\sigma_{k}<\frac{1}{2}$ and $\sigma_{k}^{\wedge}=\sigma_{k}$, so the length of the short runs on the level $k+1$ is $a_{i_{a}(k+1)}$. Because $\delta_{1}\left(a_{i_{a}(k+1)}\right)=0$, we get the statement about the run lengths.

- $a_{i_{a}(k+1)}=1$. This means that $\sigma_{k}>\frac{1}{2}$ and (from Def. 1) $\sigma_{k}^{\wedge}=1-\sigma_{k}=$ $\left[0 ; 1+a_{i_{a}(k+1)+1}, a_{i_{a}(k+1)+2}, \ldots\right]$, so the length of the short runs on the level $k+1$ is $1+a_{i_{a}(k+1)+1}=a_{i_{a}(k+1)}+\delta_{1}\left(a_{i_{a}(k+1)}\right) \cdot a_{i_{a}(k+1)+1}$.

Theorem 3 gives the statement concerning the form of runs on all levels. It says that $\operatorname{Reg}_{a}(k)=2 k-i_{a}(k+1)$ and $\operatorname{Reg}_{a}(k+1)=2 k+2-i_{a}(k+2)$, so the condition $\operatorname{Reg}_{a}(k+1)=\operatorname{Reg}_{a}(k)$ is equivalent to $i_{a}(k+2)=i_{a}(k+1)+2$, thus, 
according to Def. 3] to $\delta_{1}\left(a_{i_{a}(k+1)}\right)=1$, so $a_{i_{a}(k+1)}=1$. In the same way we show that the condition $\operatorname{Reg}_{a}(k+1)=\operatorname{Reg}_{a}(k)+1$ is equivalent to $a_{i_{a}(k+1)} \neq 1$. Moreover, because $\operatorname{Reg}_{a}(k)=2 k-i_{a}(k+1)$, the parity of $\operatorname{Reg}_{a}(k)$ and $i_{a}(k+1)$ is the same for all $k$, so we can replace " $\operatorname{Reg}_{a}(k)$ is even" from Theorem 1 by " $i_{a}(k+1)$ is even" in the CF description.

\section{Some Applications of the Main Result}

\subsection{Slopes with Periodic CF Expansions}

Period Length 1. We are looking for numbers $a \in] 0,1[\backslash \mathbb{Q}$ having periodic $\mathrm{CF}$ expansion with period length 1 , i.e., $a=[0 ; n, n, \ldots]=[0 ; \bar{n}]$. This means that $a$ is a root of equation $a=\frac{1}{n+a}$, thus of $a^{2}+n a-1=0$, which gives $a=\frac{1}{2}\left(\sqrt{n^{2}+4}-n\right)$, because $\left.a \in\right] 0,1[$. We have two groups of lines:

- when $n=1$, we get a one-element group, containing the line $y=\frac{1}{2}(\sqrt{5}-1) x$ with the Golden Section as slope. Here we have $i_{a}(1)=1$ and for $k \geq 2$ there is $i_{a}(k)=2 k-2$, which is always even. Moreover, $b_{1}=1$ and for $k \geq 1$ we have $b_{k+1}=a_{2 k}+a_{2 k+1}=2$. According to Theorem 4 we get the following digitization: $\left\|S_{1}\right\|=1,\left\|L_{1}\right\|=2$; for $k \in \mathbb{N}^{+} S_{k+1}=S_{k} L_{k}, L_{k+1}=S_{k} L_{k}^{2}$.

- when $n \geq 2$, we have $i_{a}(k)=k$ for each $k \in \mathbb{N}^{+}$and $b_{k+1}=a_{k+1}=n$ for all $k \in \mathbb{N}$. This means, according to Theorem 4, that for all the lines $y=$ $\frac{1}{2}\left(\sqrt{n^{2}+4}-n\right) x$ where $n \in \mathbb{N}^{+} \backslash\{1\}$, we get the following description of the digitization: $\left\|S_{1}\right\|=n,\left\|L_{1}\right\|=n+1$; for $k \in \mathbb{N}^{+}: S_{2 k}=S_{2 k-1}^{n-1} L_{2 k-1}, L_{2 k}=$ $S_{2 k-1}^{n} L_{2 k-1}, S_{2 k+1}=L_{2 k} S_{2 k}^{n-1}, L_{2 k+1}=L_{2 k} S_{2 k}^{n}$.

Period Length 2. Now we are looking for numbers $a \in] 0,1[\backslash \mathbb{Q}$ having periodic $\mathrm{CF}$ expansion with period length 2 , i.e., $a=[0 ; \overline{n, m}]$. This means that $a$ is a root of equation $a=[0 ; n, m+a]$, thus of $n a^{2}+m n a-m=0$, which gives $a=\frac{1}{2 n}\left(\sqrt{m^{2} n^{2}+4 m n}-m n\right)$, because $\left.a \in\right] 0,1[$. If $m=n$, see the description for period length 1 . If $m \neq n$, we get three possible classes of lines:

- when $m, n \geq 2$, we have $i_{a}(k)=k$ for each $k \in \mathbb{N}^{+}$and $b_{k+1}=a_{k+1}$ for all $k \in \mathbb{N}$. This means, from Theorem 4 that for all the lines $y=a x$, where $a=\frac{1}{2 n}\left(\sqrt{m^{2} n^{2}+4 m n}-m n\right)$ for some $n, m \in \mathbb{N}^{+} \backslash\{1\}$, we get the following description of the digitization: $\left\|S_{1}\right\|=n,\left\|L_{1}\right\|=n+1$; for $k \in \mathbb{N}^{+}: S_{2 k}=$ $S_{2 k-1}^{m-1} L_{2 k-1}, L_{2 k}=S_{2 k-1}^{m} L_{2 k-1}, S_{2 k+1}=L_{2 k} S_{2 k}^{n-1}, L_{2 k+1}=L_{2 k} S_{2 k}^{n}$.

- when $m=1$ and $n \geq 2$, we have $i_{a}(1)=1$ and $b_{1}=n$. For $k \in \mathbb{N}^{+}$there is $i_{a}(k+1)=2 k$ and $b_{k+1}=a_{2 k}+a_{2 k+1}=n+1$. The digitization is thus: $\left\|S_{1}\right\|=n,\left\|L_{1}\right\|=n+1$; for $k \in \mathbb{N}^{+}: S_{k+1}=S_{k} L_{k}^{n}, L_{k+1}=S_{k} L_{k}^{n+1}$.

- when $m \geq 2$ and $n=1$, we have $i_{a}(1)=1, i_{a}(2)=2$ and $i_{a}(k+1)=2 k-1$ for $k \geq 2$, which means that $i_{a}(k)$ is odd for all $k \neq 2$. Moreover, $b_{1}=1, b_{2}=m$ and $b_{k+1}=a_{2 k-1}+a_{2 k}=1+m$ for $k \geq 2$. The digitization is thus as follows: $\left\|S_{1}\right\|=1,\left\|L_{1}\right\|=2, S_{2}=S_{1}^{m-1} L_{1}, L_{2}=S_{1}^{m} L_{1}$, and for $k \geq 2$ we have $S_{k+1}=L_{k}^{m} S_{k}$ and $L_{k+1}=L_{k}^{m+1} S_{k}$. 
Generally - Quadratic Surds. Let us recall that an algebraic number of degree $n$ is a root of an algebraic equation $a_{0} x^{n}+a_{1} x^{n-1}+\cdots+a_{n-1} x+a_{n}=0$ of degree $n$ with integer coefficients, but is not a root of any algebraic equation of lower degree with integer coefficients. Algebraic numbers of the second degree are called quadratic irrationals or quadratic surds. The following theorem is a merge of Lagrange's theorem from 1770 with Euler's theorem from 1737 (see 1, pp. 66-71). Quadratic surds, and only they, are represented by periodic CFs, meaning purely or mixed periodic ([1, p. 66). It follows from this theorem that all the lines with quadratic surds from the interval ]0,1[ as slopes have simple digitization patterns, which can be described by general formulae for all of the digitization levels. Moreover, in [10] on p. 88, we find the following theorem.

If $d$ is a positive, non-square integer, then $\sqrt{d}=\left[x_{0} ; \overline{x_{1}, x_{2}, \ldots, x_{2}, x_{1}, 2 x_{0}}\right]$, where each partial quotient is a positive integer.

The CFs of pure quadratic irrationals all have the same structure, involving palindromes. Sequence $\mathbf{A 0 0 3 2 8 5}$ in $\left[12\right.$ shows for each $n \in \mathbb{N}^{+}$the length of the period of $\mathrm{CF}$ for $\sqrt{n}$ ( 0 if $n$ is a square). Also in [10, on p. 89, we find some patterns in the CF expansions of quadratic surds, for example $\sqrt{k^{2}+1}=[k ; \overline{2 k}]$, $\sqrt{k^{2}+2}=[k ; \overline{k, 2 k}], \sqrt{k^{2}+m}=[k ; \overline{2 k / m, 2 k}]$. These patterns make it very easy to construct the digitization of the lines with slopes $\sqrt{k^{2}+1}-k, \sqrt{k^{2}+2}-k$, or, generally, $\sqrt{k^{2}+m}-k$, using Theorem 4 from the present paper. See [10], pp. 83-91, for both theory and examples on this subject.

\subsection{Slopes with Non-periodic CF Expansions}

Quadratic irrationals are not the only numbers showing simple patterns in their CF expansion. There also exist transcendental numbers with simple patterns. CF sequences for some transcendental number have periodic forms. Some examples can be found, among others, on p. 97 in [2]. The examples were given by Euler in 1737, but the first of them was, according to Brezinski, already given by $\mathrm{R}$. Cotes in the Philosophical Transactions in 1714.

$$
\begin{gathered}
e-2=[0 ; 1,2,1,1,4,1,1,6,1, \ldots, 1,2 k, 1, \ldots]=[0 ; \overline{1,2 k, 1}]_{k=1}^{\infty}, \\
\frac{e+1}{e-1}-2=[0 ; \overline{2+4 k}]_{k=1}^{\infty}, \quad \frac{e-1}{2}=[0 ; 1, \overline{2+4 k}]_{k=1}^{\infty} .
\end{gathered}
$$

On p. 124 in [10] we find the following. For $n \geq 2$

$$
\sqrt[n]{e}-1=[0 ; \overline{(2 k-1) n-1,1,1}]_{k=1}^{\infty}
$$

On p. 110 in [2] we find the following formula, obtained by Euler in 1737 and Lagrange in 1776, but each using different methods:

$$
\frac{e^{2}-1}{e^{2}+1}=[0 ; 1,3,5, \ldots, 2 k-1, \ldots]=[0 ; \overline{2 k-1}]_{k=1}^{\infty} .
$$

This means that we are able to describe exactly, i.e., not by using approximations by rationals, the construction of the digital lines $y=a x$, where $a$ is equal to 
$e-2, \frac{e+1}{e-1}-2, \frac{e-1}{2}, \sqrt{e}-1, \sqrt[3]{e}-1$ or $\frac{e^{2}-1}{e^{2}+1}$, using Theorem 4 from the present paper. Because of the repeating pattern in the CF expansions of the slopes, we are able to obtain general formulae for all of the digitization levels.

For example, if the slope $a$ is equal to one of the following $\frac{e+1}{e-1}-2, \frac{e-1}{2}, \frac{e^{2}-1}{e^{2}+1}$, then the digitization patterns can be described for all of them in the following way. For all $k \in \mathbb{N}^{+}$there is $i_{a}(k)=k$, thus $b_{k}=a_{k}$, because there are no elements $a_{k}=1$ for $k \geq 2$ in the CF expansions. This gives the following digitization pattern for these lines: $\left\|S_{1}\right\|=a_{1},\left\|L_{1}\right\|=a_{1}+1$ and for $k \in \mathbb{N}^{+}$

$$
\left(S_{k+1}, L_{k+1}\right)= \begin{cases}\left(S_{k}^{a_{k+1}-1} L_{k},\right. & \left.S_{k}^{a_{k+1}} L_{k}\right) \text { if } k \text { is odd } \\ \left(L_{k} S_{k}^{a_{k+1}-1},\right. & \left.L_{k} S_{k}^{a_{k+1}}\right) \text { if } k \text { is even } .\end{cases}
$$

The only difference in the digitization patterns for the three slopes are different run lengths, defined by the elements $a_{k}$ of the CF expansions (14) and (16).

Formula (13) gives the digitization of the line $y=a x$ with $a=e-2$. Here $i_{a}(2 k)=3 k-1\left(a_{i_{a}(2 k)}=2 k \neq 1\right)$ and $i_{a}(2 k+1)=3 k\left(a_{i_{a}(2 k+1)}=1\right)$ for $k \in$ $\mathbb{N}^{+}$, so we get $b_{1}=1, b_{2 k}=2 k$ and $b_{2 k+1}=2$ for $k \in \mathbb{N}^{+}$, and the digitization pattern is as follows: $\left\|S_{1}\right\|=1,\left\|L_{1}\right\|=2$ and for $k \in \mathbb{N}^{+}$

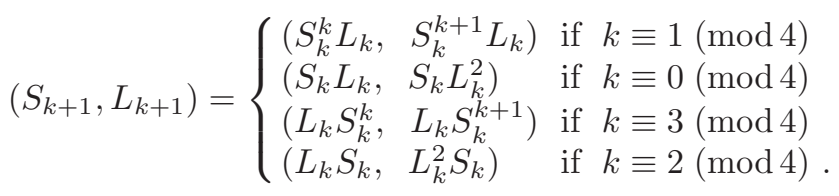

For example, $S_{5}=S_{4} L_{4}=\left(L_{3} S_{3}^{3}\right)\left(L_{3} S_{3}^{4}\right)=\left(L_{2}^{2} S_{2}\right)\left(L_{2} S_{2}\right)^{3}\left(L_{2}^{2} S_{2}\right)\left(L_{2} S_{2}\right)^{4}=$ $\left(S_{1}^{2} L_{1}\right)^{2}\left(S_{1} L_{1}\right)\left[\left(S_{1}^{2} L_{1}\right)\left(S_{1} L_{1}\right)\right]^{3}\left(S_{1}^{2} L_{1}\right)^{2}\left(S_{1} L_{1}\right)\left[\left(S_{1}^{2} L_{1}\right)\left(S_{1} L_{1}\right)\right]^{4}$, where $\left\|S_{1}\right\|=1$ and $\left\|L_{1}\right\|=2$.

\section{Conclusion}

We have presented a computationally simple description of the digitization of straight lines $y=a x$ with slopes $a \in] 0,1[\backslash \mathbb{Q}$, based on the CF expansions of the slopes. The description reflects the hierarchical structure of digitization runs. Moreover, it is exact, avoiding approximations by rationals.

The theoretical part of the paper was based on [15] and the examples were based on the literature concerning CFs ([2] and [10]). The examples show how to use the theory in finding digitization patterns. This description can also be useful in theoretical research on digital lines with irrational slopes. The present study may serve as a springboard for future research on some classes of digital lines, defined by the CF expansions of their slopes.

The new method gives a special treatment to the CF elements equal to 1 , which makes it very powerful for some slopes with 1's in the CF expansion. To our knowledge, there exist no other methods of describing digital lines with irrational slopes by $\mathrm{CF}$ s which give a special treatment to $\mathrm{CF}$ elements equal to 1, which makes our method original. 
A comparison between our new method and some other CF methods will be presented in a forthcoming paper by the author. We will show for example how to construct a slope $a \in] 0,1[\backslash \mathbb{Q}$ so that for each $n \geq 2$ the difference between the length of the digital straight line segment (its cardinality as a subset of $Z^{2}$ ) of $y=a x$ produced in the $n$th step of our method and the length of the digital straight line segment of $y=a x$ produced in the $n$th step of the method by Venkov (described in [16] on p. 67) is as large as we decide in advance.

Acknowledgments. I am grateful to Christer Kiselman for comments on earlier versions of the manuscript.

\section{References}

1. Beskin, N.M.: Fascinating Fractions. Mir Publishers, Moscow (1986) (Revised from the 1980 Russian edition)

2. Brezinski, C.: History of Continued Fractions and Padé Approximants. Springer, Heidelberg (1991) (Printed in USA)

3. Brons, R.: Linguistic Methods for the Description of a Straight Line on a Grid. Comput. Graphics Image Processing 3, 48-62 (1974)

4. Bruckstein, A.M.: Self-Similarity Properties of Digitized Straight Lines. Contemp. Math. 119, 1-20 (1991)

5. Debled, I.: Étude et reconnaissance des droites et plans discrets. Ph.D. Thesis, Strasbourg: Université Louis Pasteur, pp. 209 (1995)

6. Dorst, L., Duin, R.P.W.: Spirograph Theory: A Framework for Calculations on Digitized Straight Lines. IEEE Transactions on Pattern Analysis and Machine Intelligence PAMI 6(5), 632-639 (1984)

7. Khinchin, A.Ya.: Continued Fractions, 3rd edn. Dover Publications (1997)

8. Klette, R., Rosenfeld, A.: Digital straightness - a review. Discrete Appl. Math. 139(1-3), 197-230 (2004)

9. Lothaire, M.: Algebraic Combinatorics on Words. Cambridge Univ. Press, Cambridge (2002)

10. Perron, O.: Die Lehre von den Kettenbrüchen. Band I: Elementare Kettenbrüche, 3rd edn (1954)

11. Réveillès, J.-P.: Géométrie discrète, calculus en nombres entiers et algorithmique, 251 pages, Strasbourg: Université Louis Pasteur, Thèse d'État (1991)

12. Sloane, N.J.A.: The On-Line Encyclopedia of Integer Sequences, http://www.research.att.com/ njas/sequences/A003285

13. Stephenson, P.D.: The Structure of the Digitised Line: With Applications to Line Drawing and Ray Tracing in Computer Graphics. North Queensland, Australia, James Cook University. Ph.D. Thesis (1998)

14. Stolarsky, K.B.: Beatty sequences, continued fractions, and certain shift operators. Canad. Math. Bull. 19, 473-482 (1976)

15. Uscka-Wehlou, H.: Digital lines with irrational slopes. Theoret. Comput. Sci. 377, 157-169 (2007)

16. Venkov, B.A.: Elementary Number Theory. Translated and edited by Helen Alderson. Wolters-Noordhoff, Groningen (1970)

17. de Vieilleville, F., Lachaud, J.-O.: Revisiting Digital Straight Segment Recognition. In: Kuba, A., Nyúl, L.G., Palágyi, K. (eds.) DGCI 2006. LNCS, vol. 4245, pp. 355366. Springer, Heidelberg (2006)

18. Voss, K.: Discrete Images, Objects, and Functions in $\mathbb{Z}^{n}$. Springer-Verlag (1993) 